\title{
On the Estimations of the Small Periodic Eigenvalues
}

\author{
Seza Dinibütün and O. A. Veliev \\ Department of Mathematics, Dogus University, Acıbadem, Kadiköy, 81010 Istanbul, Turkey
}

Correspondence should be addressed to Seza Dinibütün; sdinibutun@dogus.edu.tr

Received 15 April 2013; Revised 13 June 2013; Accepted 16 June 2013

Academic Editor: Ferhan M. Atici

Copyright (C) 2013 S. Dinibütün and O. A. Veliev. This is an open access article distributed under the Creative Commons Attribution License, which permits unrestricted use, distribution, and reproduction in any medium, provided the original work is properly cited.

We estimate the small periodic and semiperiodic eigenvalues of Hill's operator with sufficiently differentiable potential by two different methods. Then using it we give the high precision approximations for the length of $n$th gap in the spectrum of HillSehrodinger operator and for the length of $n$th instability interval of Hill's equation for small values of $n$. Finally we illustrate and compare the results obtained by two different ways for some examples.

\section{Introduction}

Let $P(q)$ and $S(q)$ be the operators generated in $L_{2}[0, \pi]$ by the differential expression

$$
-y^{\prime \prime}(x)+q(x) y(x)
$$

with the periodic

$$
y(\pi)=y(0), \quad y^{\prime}(\pi)=y^{\prime}(0)
$$

and semiperiodic

$$
y(\pi)=-y(0), \quad y^{\prime}(\pi)=-y^{\prime}(0)
$$

boundary conditions, respectively, where $q$ is a real periodic function with period $\pi$. The eigenvalues of $P(q)$ and $S(q)$ for $q=0$ are $(2 n)^{2}$ and $(2 n+1)^{2}$ for $n \in \mathbb{Z}$, respectively. All eigenvalues of $P(0)$ and $S(0)$, except 0 , are doubled. The eigenvalues of the operators $P(q)$ and $S(q)$, called periodic and semiperiodic eigenvalues, are denoted by $\lambda_{2 n}$ and $\lambda_{2 n+1}$ for $n \in \mathbb{Z}$, respectively, where

$$
\begin{aligned}
\lambda_{0}(q) & <\lambda_{-1}(q) \leq \lambda_{1}(q)<\lambda_{-2}(q) \leq \lambda_{2}(q) \\
& <\lambda_{-3}(q) \leq \lambda_{3}(q)<\lambda_{-4}(q) \leq \lambda_{4}(q) \cdots
\end{aligned}
$$

[1, see page 27]. The spectrum $\sigma(T(q))$ of the operator $T(q)$ generated in $L_{2}[0,2 \pi]$ by $(1)$ and the boundary conditions

$$
y(2 \pi)=y(0), \quad y^{\prime}(2 \pi)=y^{\prime}(0)
$$

is the union of the periodic and semiperiodic eigenvalues, that is,

$$
\begin{gathered}
\sigma(P)=\left\{\lambda_{2 n}: n \in \mathbb{Z}\right\}, \\
\sigma(S)=\left\{\lambda_{2 n+1}: n \in \mathbb{Z}\right\}, \quad \sigma(T)=\left\{\lambda_{n}: n \in \mathbb{Z}\right\},
\end{gathered}
$$

since (5) holds if and only if either (2) or (3) holds [1, see page 33].

The spectrum of the operator $L(q)$ generated in $L_{2}(-\infty, \infty)$ by $(1)$ consists of the intervals $\left[\lambda_{n-1}(q), \lambda_{-n}(q)\right]$ for $n=1,2, \ldots$. Moreover, these intervals are the closure of the stable intervals of equation

$$
-y^{\prime \prime}(x)+q(x) y(x)=\lambda y(x) .
$$

The intervals $\left(\lambda_{-n}, \lambda_{n}\right)$ for $n=1,2, \ldots$ are the gaps in the spectrum. These intervals with $\left(-\infty, \lambda_{0}\right)$ are the instable intervals of (7) [1, see pages 32 and 82]. The length of $n$th gap in the spectrum of $L(q)$ (the length of $(n+1)$ th instability interval of (7)) is

$$
\gamma_{n}(q)=: \lambda_{n}(q)-\lambda_{-n}(q) .
$$

Therefore the estimations of the periodic and semiperiodic eigenvalues are also the investigations of the spectrum of $L(q)$ and of the stable intervals of (7).

In this paper we gave the estimations for the small periodic and semiperiodic eigenvalues when the real periodic 
potential $q$ belongs to the Sobolev space $W_{1}^{k}[0, \pi]$ with $k>1$. These assumptions on the potential $q$ imply that

$$
q(x)=\sum_{n \in \mathbb{Z}} q_{n} e^{i 2 n x}, \quad q_{-n}=\overline{q_{n}}, \quad\left|q_{n}\right| \leq \frac{r}{(2 n)^{m}},
$$

where

$$
\begin{gathered}
q_{n}=\left(q, e^{i 2 n x}\right)=\int_{0}^{\pi} q(x) e^{-i 2 n x} d x, \\
r=\int_{[0, \pi]}\left|q^{(k)}(x)\right| d x .
\end{gathered}
$$

Without loss of generality, it is assumed that $q_{0}=0$.

It is wellknown that (see [2])

$$
\begin{gathered}
\left|\lambda_{n}(q)-\lambda_{n}(0)\right| \leq \sup |q(x)|, \\
\lambda_{n}(0)=n^{2}, \quad \forall n \in \mathbb{Z} .
\end{gathered}
$$

To give a subtle estimate for the eigenvalues $\lambda_{n}(q)$, we write the potential $q$ in the form

$$
q(x)=p(x)+\sum_{|n|>s} q_{n} e^{i 2 n x}
$$

where

$$
p(x)=\sum_{n:|n| \leq s} q_{n} e^{i 2 n x}
$$

The inequality in (9) implies that

$$
\sup _{x \in[0, \pi]}|q(x)-p(x)| \leq \sum_{|n|>s}\left|q_{n}\right| \leq \frac{r}{(k-1)(2 s)^{k-1}} .
$$

Hence, by the perturbation theory (see [2]) we have

$$
\begin{aligned}
& \left|\lambda_{n}(q)-\lambda_{n}(p)\right| \leq \frac{r}{(k-1)(2 s)^{k-1}} \\
& \left|\gamma_{n}(q)-\gamma_{n}(p)\right| \leq \frac{2 r}{(k-1)(2 s)^{k-1}}
\end{aligned}
$$

Therefore to estimate $\lambda_{n}(q)$ and $\gamma_{n}(q)$ we can investigate the eigenvalues $\lambda_{n}(p)$ of the operator $T(p)$ and then use (8) and (15).

In the literature, there are a lot of studies about numerical estimation of the periodic and semiperiodic eigenvalues by using the finite difference method, finite element method, Prüfer transformations, and shooting method. Let us recall some of them. Andrew considered the computations of the eigenvalues by using finite element method [3] and finite difference method [4]. Then these results have been extended by Condon [5] and by Vanden Berghe et al. [6]. Ji and Wong used Prüfer transformation and shooting method in their studies [7-9]. Malathi et al. [10] used shooting technique and direct integration method for computing eigenvalues of periodic Sturm-Liouville problems.

We consider the small periodic and semiperiodic eigenvalues by other methods. First, in Section 2, we obtain an approximation of the eigenvalues $\lambda_{ \pm n}(p)$ for $n>m s$, where $m$ is the positive integer for determination of the error in estimations, by using the method of the paper [11], where the asymptotic formulas for the eigenvalues and eigenfunctions of the $t$-periodic boundary value problems were obtained. Then, in Section 3, using it and considering the matrix form of $T(p)$ we give an approximation with very small errors for all small periodic and semiperiodic eigenvalues. Finally, we apply these investigations to get approximations order $10^{-18}, 10^{-15}$, and $10^{-12}$ for the first 201 eigenvalues of the operator $T$ with potentials $p_{1}(x)=2 \cos 2 x, p_{2}(x)=$ $2 \cos 2 x+2 \cos 4 x$, and $p_{3}(x)=2 \cos 2 x+2 \cos 4 x+2 \cos 6 x$, respectively, and give a comparison between the approximated eigenvalues obtained by the different ways.

\section{On Applications of the Asymptotic Methods}

In this and next sections, for simplicity of the notation, $\lambda_{n}(p)$ is denoted by $\lambda_{n}$. By (11)-(13)

$$
\left|\lambda_{n}-n^{2}\right| \leq \sup |p(x)| \leq \sum_{n=-s}^{s}\left|q_{n}\right| .
$$

To get the subtle estimations for $\lambda_{n}$, that is, to observe the influence of the trigonometric polynomial $p(x)$ to the eigenvalue $n^{2}$ of $T(0)$, we use the formula

$$
\left(\lambda_{N}-n^{2}\right)\left(\Psi_{N}, e^{i n x}\right)=\left(p \Psi_{N}, e^{i n x}\right)
$$

obtained from the equation

$$
-\Psi_{N}^{\prime \prime}(x)+p(x) \Psi_{N}(x)=\lambda_{N} \Psi_{N}(x)
$$

by multiplying $e^{i n x}$, where $\Psi_{N}$ is the eigenfunction corresponding to the eigenvalue $\lambda_{n} ;\left\|\Psi_{n}\right\|=1 / \sqrt{\pi},(\cdot, \cdot)$ and $\|\cdot\|$ denote inner product and norm in $L_{2}[0, \pi]$.

Introduce the notation

$$
\begin{gathered}
M=\sup _{x}|p(x)|, \quad c=\sum_{n=-s}^{s}\left|q_{n}\right|, \\
Q=\sup _{n}\left|q_{n}\right|, \quad X_{N, n}=\left(\Psi_{N}, e^{i n x}\right) .
\end{gathered}
$$

Using this notation and (13) in (17) we get

$$
\left(\lambda_{N}-n^{2}\right) X_{N, n}=\sum_{k=-s}^{s} q_{k} X_{N, n-2 k}
$$

In (20) replacing $N$ by $n$ and then iterating it $m$ times, as in the paper [11], were done; we obtain

$$
\left(\lambda_{n}-n^{2}\right) X_{n, n}=A_{m}\left(\lambda_{n}, n\right) X_{n, n}+R_{m+1}\left(\lambda_{n}, n\right),
$$


where

$$
A_{m}\left(\lambda_{n}, n\right)=\sum_{k=1}^{m} a_{k}\left(\lambda_{n}, n\right)
$$

$$
\begin{aligned}
& a_{k}\left(\lambda_{n}, n\right) \\
& =\sum_{n_{1}, n_{2}, \ldots, n_{k}=-s}^{s} \frac{q_{n_{1}} q_{n_{2}} \cdots q_{n_{k}} q_{-n_{1}-n_{2}-\cdots-n_{k}}}{\prod_{i=1,2, \ldots, k}\left[\lambda_{n}-\left(n-2 n_{1}-2 n_{2} \cdots-2 n_{i}\right)^{2}\right]}, \\
& R_{m+1}\left(\lambda_{n}, n\right) \\
& \quad \sum_{n_{1}, n_{2}, \ldots, n_{m+1}=-s}^{s} \\
& \quad \times \frac{q_{n_{1}} q_{n_{2}} \cdots q_{n_{m}} q_{n_{m+1}} X_{n, n-2 n_{1}-2 n_{2}-\cdots-2 n_{m+1}}}{\prod_{i=1,2, \ldots m}\left[\lambda_{n}-\left(n-2 n_{1}-2 n_{2} \cdots-2 n_{i}\right)^{2}\right]}, \\
& \quad n_{j} \neq 0, \quad \forall j, \sum_{j=1}^{k} n_{j} \neq 0, \quad \forall k=1,2, \ldots, m
\end{aligned}
$$

under assumption that

$$
\lambda_{n}-\left(n-2 n_{1} \cdots-2 n_{i}\right)^{2} \neq 0
$$

for $i=1,2, \ldots, m$. Now using (21), estimating $X_{n, n}$ and $R_{m+1}$, we prove the following,

Theorem 1. Let $m$ be a positive integer. If the conditions

$$
|n|>m s, \quad 4(|n|-1) \geq 3 M
$$

hold, then the eigenvalue $\lambda_{n}$ of the operator $T(p)$ satisfies

$$
\begin{aligned}
\lambda_{n}= & n^{2} \\
& +\sum_{k=1}^{m} \sum_{n_{1}, n_{2}, \ldots, n_{k}=-s}^{s} \\
& \times \frac{q_{n_{1}} q_{n_{2}} \cdots q_{n_{k}} q_{-n_{1}-n_{2}-\cdots-n_{k}}}{\prod_{i=1,2, \ldots k}\left[\lambda_{n}-\left(n-2 n_{1}-2 n_{2} \cdots-2 n_{i}\right)^{2}\right]} \\
& +\alpha_{n, m},
\end{aligned}
$$

where

$$
\left|\alpha_{n, m}\right| \leq \frac{2 c^{m+1}}{(4(|n|-1)-M)^{m}} ;
$$

$c, M$, and $p(x)$ are defined in (19) and (13).

Proof. Since $q_{0}=0$ we have $0<\left|n_{i}\right| \leq s$. This with (16), (19), and (26) implies that

$$
\begin{aligned}
\mid \lambda_{n}- & \left(n-2 n_{1}-2 n_{2}-\cdots-2 n_{i}\right)^{2} \mid \\
& \geq\left|n^{2}-(|n|-2)^{2}\right|-M \\
& =4(|n|-1)-M \geq 2 M>0
\end{aligned}
$$

for $i=1,2, \ldots, m$; that is, assumption (25) holds. Therefore we can use (21).

Now we estimate $X_{n, n}$ and $R_{m+1}$. First let us estimate $R_{m+1}$. Since $\left\|\Psi_{n}\right\|=1 / \sqrt{\pi}$ by Schwarz inequality we have

$$
\left|\left(\Psi_{n}(x), e^{i\left(n-2 n_{1}-2 n_{2}-\cdots-2 n_{m+1}\right) x}\right)\right| \leq 1 .
$$

This with (23) and (29) implies that

$$
\begin{aligned}
\left|R_{m+1}\right| \leq & \frac{1}{(4(|n|-1)-M)^{m}} \\
& \times \sum_{n_{1}, n_{2}, \ldots, n_{m+1}=-s}^{s}\left|q_{n_{1}} q_{n_{2}} \cdots q_{n_{m}} q_{n_{m+1}}\right| .
\end{aligned}
$$

Hence by definition of $c$ (see (19)) we have

$$
\left|R_{m+1}\right| \leq \frac{c^{m+1}}{(4(|n|-1)-M)^{m}} .
$$

Now we estimate $X_{n, n}$. Arguing as in the proof of (29) we get

$$
\left|\lambda_{n}-(n-2 k)^{2}\right| \geq 2 M, \quad \forall k \neq 0, n .
$$

Therefore using (17) we get

$$
\begin{aligned}
\sum_{k \in \mathbb{Z}, k \neq 0, n}\left|X_{n, n-2 k}\right|^{2} & =\sum_{k \in \mathbb{Z}, k \neq 0, n} \frac{\left|\left(\Psi_{n}, p e^{i((n-2 k)) x}\right)\right|^{2}}{\left|\lambda_{n}-(n-2 k)^{2}\right|^{2}} \\
& \leq \frac{M^{2}}{(2 M)^{2}}=\frac{1}{4} .
\end{aligned}
$$

This with Parseval's equality

$$
\sum_{k \in \mathbb{Z}}\left|X_{n, n-2 k}\right|^{2}=\sum_{k \in \mathbb{Z},}\left|\left(\Psi_{n}, e^{i((n-2 k)) x}\right)\right|^{2}=1
$$

implies that

$$
\left|X_{n, n}\right|^{2}+\left|X_{n,-n}\right|^{2} \geq \frac{3}{4}
$$

Hence at least one of the inequalities

$$
\left|X_{n, n}\right| \geq \frac{1}{2}, \quad\left|X_{n,-n}\right| \geq \frac{1}{2}
$$

holds. If the first inequality holds, then dividing both sides of (21) by $X_{n, n}$ and using (23), (32) we obtain the proof of (27) and (28). If the second inequality holds, then instead of (21) using

$$
\left(\lambda_{n}-(-n)^{2}\right) X_{n,-n}=A_{m}\left(\lambda_{n},-n\right) X_{n,-n}+R_{m+1}\left(\lambda_{n},-n\right),
$$

taking into account that $A_{m}\left(\lambda_{n},-n\right)=A_{m}\left(\lambda_{n}, n\right)$ and arguing as in the first case we get the proof in the second case. Theorem is proved. 
Now using (27) let us show that $\lambda_{ \pm n}$ is close to the root of the equation

$$
x=n^{2}+f(x)
$$

where

$$
\begin{aligned}
f(x)= & \sum_{n_{1}=-s}^{s} \frac{q_{n_{1}} q_{-n_{1}}}{x-\left(n-2 n_{1}\right)^{2}} \\
& +\sum_{n_{1}, n_{2}=-s}^{s} \frac{q_{n_{1}} q_{n_{2}} q_{-n_{1}-n_{2}}}{\left(x-\left(n-2 n_{1}\right)^{2}\right)\left(x-\left(n-2 n_{1}-2 n_{2}\right)^{2}\right)} \\
& +\ldots+\sum_{n_{1}, n_{2}, \ldots n_{m}=-s}^{s} \\
& \times\left(\left(q_{n_{1}} q_{n_{2}} \cdots q_{n_{m}} q_{-n_{1}-n_{2}-\cdots-n_{m}}\right)\right. \\
& \quad \times\left(\left[x-\left(n-2 n_{1}\right)^{2}\right]\left[x-\left(n-2 n_{1}-2 n_{2}\right)^{2}\right]\right. \\
& \left.\left.\quad \cdots\left[x-\left(n-2 n_{1}-2 n_{2}-\cdots-2 n_{m}\right)^{2}\right]\right)^{-1}\right) .
\end{aligned}
$$

Theorem 2. Let $n$ be a positive integer satisfying

$$
n>m s, \quad 4(n-1)>M+2 c .
$$

Then for all $x$ and $y$ from $\left[n^{2}-M, n^{2}+M\right]$ the inequality

$$
|f(x)-f(y)|<K_{n}|x-y|
$$

where

$$
K_{n}=\frac{Q c}{(4(n-1)-M)(4(n-1)-M-c)}<\frac{1}{2},
$$

holds, and (39) has a unique solution $r_{n}$ on $\left[n^{2}-M, n^{2}+M\right]$. Moreover

$$
\left|\lambda_{ \pm n}-r_{n}\right|<\frac{2 c^{m+1}}{\left(1-K_{n}\right)(4(n-1)-M)^{m}}
$$

and the length $\gamma_{n}$ of $n$th gap in the spectrum of $L(p)$ (the length $\gamma_{n}$ of $(n+1)$ th instability interval of $\left.(7)\right)$ satisfies

$$
\gamma_{n}=\lambda_{n}-\lambda_{-n}<\frac{4 c^{m+1}}{\left(1-K_{n}\right)(4(n-1)-M)^{m}} .
$$

Proof. Let $f_{1}(x), f_{2}(x), \ldots, f_{m}(x)$ be the first, second, and $m$ th summations in the right-hand side of (40). Then

$$
f_{1}^{\prime}(x)=-\sum_{k=-s}^{s} \frac{\left|q_{k}^{2}\right|}{\left(x-(n-2 k)^{2}\right)^{2}} .
$$

For $x \in\left[n^{2}-M, n^{2}+M\right]$, using (29) and (41), we get

$$
\left|x-(n-2 k)^{2}\right| \geq 4(n-1)-M>2 c .
$$

On the other hand

$$
\sum_{k=-s}^{s}\left|q_{k}^{2}\right| \leq Q c .
$$

This inequality with (47) and the inequality $Q \leq c$ (see (19)) imply that

$$
\left|f_{1}^{\prime}(x)\right| \leq \frac{Q c}{(4(n-1)-M)^{2}}<\frac{1}{4} .
$$

In the same way we obtain

$$
\left|f_{k}^{\prime}(x)\right| \leq \frac{Q c^{k}}{(4(n-1)-M)^{k+1}}<\frac{1}{2^{k+1}}
$$

for $k=2,3, \ldots$ Thus by the geometric series formula we have

$$
\left|f^{\prime}(x)\right| \leq K_{n}<\frac{1}{2}, \quad \forall x \in\left[n^{2}-M, n^{2}+M\right],
$$

where $K_{n}$ is defined in (43), and by mean-value theorem (42) holds. Therefore by contraction mapping theorem (39) has a unique solution $r_{n}$ on $\left[n^{2}-M, n^{2}+M\right]$.

Now let us prove (44). Let $F(x)=x-n^{2}-f(x)$. Using the definition of $r_{n}$ and $F(x)$ and then (40) we obtain $F\left(r_{n}\right)=0$ and

$$
\left|F\left(\lambda_{n}\right)-F\left(r_{n}\right)\right| \leq\left|\alpha_{n, m}\right| \text {. }
$$

On the other hand by (51) we have $\left|F^{\prime}(x)\right| \geq 1-K_{n}$ for all $x \in\left[n^{2}-M, n^{2}+M\right]$. Therefore using the mean-value formula

$$
\left|F\left(\lambda_{n}\right)-F\left(r_{n}\right)\right|=\left|F^{\prime}(\zeta)\right|\left|\lambda_{n}-r_{n}\right|,
$$

$\zeta \in\left[n^{2}-M, n^{2}+M\right]$, and (52) we obtain

$$
\left|\lambda_{n}-r_{n}\right| \leq \frac{\left|\alpha_{n, m}\right|}{1-K_{n}}
$$

This with (28) implies (44) for $\lambda_{n}$. In the same way we prove (44) for $\lambda_{-n}$. Therefore (45) follows from (44). The theorem is proved.

Now let us approximate $r_{n}$ by fixed-point iteration

$$
x_{n, 0}=n^{2}, \quad x_{n, 1}=n^{2}+f\left(x_{n, 0}\right), \ldots, x_{n, i}=n^{2}+f\left(x_{n, i-1}\right) .
$$

Note that repeating the proof of (51) one can readily see that

$$
\left|f\left(\lambda_{n}\right)\right| \leq \frac{Q c}{4(n-1)-M-c}, \quad\left|f\left(n^{2}\right)\right| \leq \frac{Q c}{4(n-1)-c}
$$

for all $n$ satisfying (41).

Theorem 3. For the sequence $\left\{x_{n, i}\right\}$ defined by (55) the estimations

$$
\left|x_{n, i}-r_{n}\right| \leq K_{n}^{i} B
$$

for $i=1,2,3, \ldots$ hold, where $n$ satisfies (41), $K_{n}$ is defined in Theorem 2, and

$$
B=\frac{\left|f\left(n^{2}\right)\right|}{1-K_{n}} \leq \frac{Q c}{\left(1-K_{n}\right)(4(n-1)-c)} .
$$


Proof. It is clear and well known that if $f$ satisfies (42) then

$$
\left|x_{n, i}-r_{n}\right| \leq K_{n}^{i}\left|x_{n, 0}-r_{n}\right| \text {. }
$$

Therefore to prove (57) it is enough to show that

$$
\left|x_{n, 0}-r_{n}\right| \leq B,
$$

where $B$ is defined in (58). By definition of $r_{n}$ and $x_{n, 0}$ we have

$$
r_{n}-x_{n, 0}=f\left(r_{n}\right)=f\left(r_{n}\right)-f\left(x_{n, 0}\right)+f\left(n^{2}\right),
$$

and by the mean-value theorem there exists $x \in\left[n^{2}-M, n^{2}+\right.$ $M]$ such that

$$
f\left(r_{n}\right)-f\left(x_{n, 0}\right)=f^{\prime}(x)\left(r_{n}-x_{n, 0}\right) .
$$

These two equalities imply that

$$
\left(r_{n}-x_{n, 0}\right)\left(1-f^{\prime}(x)\right)=f\left(n^{2}\right) .
$$

This formula with (56) and (51) implies (60).

Thus by (44) and (57) we have the approximation $x_{n, i}$ for $\lambda_{ \pm n}$ with the error

$$
E_{n, i}=:\left|\lambda_{ \pm n}-x_{n, i}\right|<\frac{2 c^{m+1}}{\left(1-K_{n}\right)(4(n-1)-M)^{m}}+K_{n}^{i} B .
$$

\section{Estimation of the Small Eigenvalues}

In this section we estimate the eigenvalues $\lambda_{N}$ of the operator $T(p)$, for $|N| \leq l$, by investigating the system of $2 S+1$ equations

$$
\begin{gathered}
\left(\lambda_{N}-n^{2}\right) X_{N, n}-\sum_{k:|k| \leq s,|n-2 k| \leq S} q_{k} X_{N, n-2 k} \\
=\sum_{k:|k| \leq s,|n-2 k|>S} q_{k} X_{N, n-2 k}
\end{gathered}
$$

for $n=-S,-S+1,-S+2, \ldots, S$, where $S=l+2 r s$ and $r$ is the positive integer for determination of the error in estimation,

$$
4(l-1)-M-c>\max \left\{c, 2 c^{2}\right\} ;
$$

the numbers $M$ and $c$ are defined in (19). The first, second, and $j$ th equations of (65) are obtained from (20) by taking $n=-S, n=-S+1$, and $n=-S-1+j$, respectively, and by writing the terms with multiplicand $X_{N, n-2 k}$ for $|n-2 k| \leq S$ on the left-hand side and the terms with multiplicand $X_{N, n-2 k}$ for $|n-2 k|>S$ on the right-hand side.

To write (65) in the matrix form let us introduce the notations. Let $A$ be $(2 S+1)$ by $(2 S+1)$ matrix $\left(a_{i, j}\right)$ defined by

$$
a_{i, i}=(-S-1+i)^{2}, \quad a_{i, i \mp 2 k}=q_{ \pm k}
$$

for $i=1,2, \ldots, 2 S+1$ and $k=1,2, \ldots s$ if $|i \mp 2 k| \leq$ $S$ and all other entries of $A$ are zero. Since $q_{-n}=\overline{q_{n}}$ (see (9)), $A$ is a Hermitian (self-adjoint) matrix and its eigenvalues are real numbers. Denote the eigenvalues of $A$ by $\mu_{0}, \mu_{-1}, \mu_{1}, \mu_{-2}, \mu_{2}, \ldots, \mu_{-S}, \mu_{S}$, where

$$
\mu_{0} \leq \mu_{-1} \leq \mu_{1} \leq \mu_{-2} \leq \mu_{2} \leq \cdots \leq \mu_{-S} \leq \mu_{S}
$$

It is clear that

$$
\left|\mu_{ \pm n}-n^{2}\right| \leq c
$$

since the diagonal elements of $A$ are $n^{2}$ for $n=-S,-S+1,-S+$ $2, \ldots, S$ and the sum of the absolute values of the nondiagonal elements of each row is not greater than $c$ (see (19)). Let $X_{N}=$ $\left(X_{N,-S}, X_{N,-S+1}, \ldots, X_{N, S}\right)$ and $R\left(\lambda_{\mathrm{N}}\right)=\left(R_{-S}, R_{-S+1}, \ldots, R_{S}\right)$ be vectors of $\mathbb{C}^{2 S+1}$, where $R_{n}=0$ for $|n| \leq S-2 s$ and

$$
R_{n}\left(\lambda_{N}\right)=\sum_{k:|k| \leq s,|n-2 k|>S} q_{k} X_{N, n-2 k}
$$

for $S-2 s<|n| \leq S$. In this notation the system of (65) can be written in the matrix form

$$
\left(\lambda_{N} I-A\right) X_{N}^{T}=R^{T}\left(\lambda_{N}\right)
$$

First we prove that $X_{N, n}$ for $n= \pm(S+1), \pm(S+2), \ldots, \pm(S+$ $2 s$ ), that is, the right-hand side $R^{T}\left(\lambda_{N}\right)$ of (71), is small (see Lemma 4). Then using it we prove that the $n$th eigenvalue $\lambda_{n}$ of the operator $T(p)$ is close to the $n$th eigenvalue $\mu_{n}$ of the matrix $A$ (see Theorem 6).

Lemma 4. If $|N| \leq l$ and $l+2 r s<|n| \leq l+2(r+1) s$, then

$$
\begin{gathered}
\left|X_{N, n}\right| \leq \frac{c^{r+1}}{(2 l)^{r+1}}=: \varepsilon, \\
\sum_{n:|n|>S}\left|X_{N, n}\right|^{2} \leq \frac{4 s \varepsilon^{2}(2 l)^{2}}{\left((2 l)^{2}-c^{2}\right)}=\frac{4 s c^{2 r+2}}{(2 l)^{2 r}\left((2 l)^{2}-c^{2}\right)}=: \delta .
\end{gathered}
$$

Proof. First we prove (72) for positive $n$. The proof for negative $n$ is similar. One can readily see from the estimations (27), (28) for $m=2,(56)$, and (66) that if $k \geq l$, then

$$
\begin{aligned}
\left|\lambda_{k}-k^{2}\right| & \leq\left|f\left(\lambda_{k}\right)\right|+\left|\alpha_{k, 2}\right| \\
& \leq \frac{Q c}{4(k-1)-M-c}+\frac{2 c^{3}}{(4(k-1)-M)^{2}}<1 .
\end{aligned}
$$

Using (74) and taking into account the condition on $N$ and $n$ we obtain

$$
\begin{aligned}
\mid \lambda_{N}- & \left(n-2 n_{1}-\cdots-2 n_{i}\right)^{2} \mid \\
& \geq\left|\lambda_{N}-(l+1)^{2}\right| \\
& \geq\left|\lambda_{l}-(l+1)^{2}\right|>\left|l^{2}-(l+1)^{2}\right|-1 \geq 2 l
\end{aligned}
$$


for $\left|n_{i}\right| \leq s, i=0,1, \ldots, r$. On the other hand iterating (20) $r$ times we get

$$
\begin{aligned}
X_{N, n}=\sum_{n_{1}, n_{2}, \ldots, n_{r}=-s}^{s} & \\
\times & \left(\left(q_{n_{1}} q_{n_{2}} \cdots q_{n_{r+1}}\left(\Psi_{N}, e^{i\left(n-2 n_{1}-\cdots-2 n_{2 r+1}\right) x}\right)\right)\right. \\
& \times\left(\left[\lambda_{N}-n^{2}\right]\left[\lambda_{N}-\left(n-2 n_{1}\right)^{2}\right]\right. \\
& \left.\left.\cdots\left[\lambda_{N}-\left(n-2 n_{1}-\cdots-2 n_{r}\right)^{2}\right]\right)^{-1}\right) .
\end{aligned}
$$

Therefore arguing as in the proof of (32) we get

$$
\left|X_{N, n}\right| \leq \frac{c^{r+1}}{(2 l)^{r+1}}
$$

for $l+2 r s<|n| \leq l+2(r+1) s$; that is, (72) is proved.

Now we prove (73). By definition of $S$ the left-hand side of (73) can be written in the form

$$
\sum_{n:|n|>S}\left|X_{N, n}\right|^{2}=\sum_{k=r}^{\infty} H_{N, k}
$$

where

$$
H_{N, k}=\sum_{l+2 k s<|n| \leq l+2(k+1) s,}\left|X_{N, n}\right|^{2} .
$$

In (72) replacing $r$ by $k$ one can readily see that

$$
H_{N, k} \leq \frac{4 s c^{2 k+2}}{(2 l)^{2 k+2}} .
$$

Using this in (78) we obtain

$$
\sum_{n:|n|>S}\left|X_{N, n}\right|^{2} \leq \sum_{k=r}^{\infty} \frac{4 s c^{2 k+2}}{(2 l)^{2 k+2}}
$$

which implies (73), since the series in the right-hand side of (81) is a geometric series with first term $4 s \varepsilon^{2}$ and factor $c^{2} /(2 l)^{2}$.

Note that (72) and (73) imply the following inequalities. By (70) and (72)

$$
\left|R_{n}\left(\lambda_{N}\right)\right|<c \varepsilon, \quad \forall n: S-2 s<|n| \leq S, \quad \forall|N| \leq l,
$$

and by the definition of $R\left(\lambda_{N}\right)$ we have

$$
\left\|R\left(\lambda_{N}\right)\right\| \leq 2 c \varepsilon \sqrt{s}, \quad \forall|N| \leq l .
$$

Besides using (73) and Parseval's equality (35) we obtain

$$
\begin{gathered}
1-\delta \leq \sum_{n=-S}^{S}\left|X_{N, n}\right|^{2} \leq 1, \\
\sqrt{1-\delta} \leq\left\|X_{N}\right\| \leq 1, \quad \forall|N| \leq l .
\end{gathered}
$$

Let $\left\{V_{n}^{T}: n=0, \pm 1, \pm 2, \ldots, \pm S\right\}$ be orthonormal system of eigenvectors of the matrix $A$ :

$$
A V_{n}^{T}=\mu_{n} V_{n}^{T}
$$

where $\left\langle V_{n}, V_{k}\right\rangle=\delta_{n, k}, V_{n}=\left(V_{n,-S}, V_{n,-S+1}, \ldots, V_{n, S}\right) \in \mathbb{C}^{2 S+1}$, and $\langle\cdot, \cdot\rangle$ denotes the inner product in $\mathbb{C}^{2 S+1}$ as well as in $l_{2}$. Denote by $D$ the $(2 S+1) \times(2 S+1)$ diagonal matrix with diagonal elements

$$
d_{i}=a_{i, i}=(-S-1+i)^{2}
$$

for $i=1,2, \ldots, 2 S+1$. The eigenfunctions of $D$ corresponding to the eigenvalues $n^{2}$ are $e_{-n}$ and $e_{n}$, where $e_{n}=$ $\left(e_{n,-S}, e_{n,-S+1}, \ldots, e_{n, S}\right)^{T}, e_{n, n}=1$, and $e_{n, k}=0$ for all $k \neq n$. Multiplying both sides of (85) for $n=N$ by $e_{n}$ we get

$$
\left(\mu_{N}-n^{2}\right) V_{N, n}=\sum_{k=-s}^{s} q_{k} V_{N, n-2 k}
$$

where $V_{N, n-2 k}=0$ if $|n-2 k|>S$. Instead of (20) using (87) and repeating the proof of (72) we obtain that if $|N| \leq l$ and $|n|>S-2 s$, then

$$
\left|V_{N, n}\right| \leq \frac{c^{r}}{(2 l)^{r}}
$$

To prove the main result of the paper we use the following.

Lemma 5. Let $c_{n, j}=\left\langle X_{n}^{T}, V_{j}^{T}\right\rangle$ and $n=0, \pm 1, \pm 2, \ldots, \pm l$. Then

$$
\left|c_{n, j}\left(\mu_{j}-\lambda_{n}\right)\right| \leq 8 s l \varepsilon^{2}
$$

for $j=0, \pm 1, \pm 2, \ldots, \pm l$ and

$$
\left|c_{n, j}\left(\mu_{j}-\lambda_{n}\right)\right| \leq 2 c \varepsilon \sqrt{s}
$$

for $j= \pm(l+1), \pm(l+2), \ldots, \pm S$.

Proof. Since $\left\{V_{j}: j=0, \pm 1, \pm 2, \ldots, \pm S\right\}$ is an orthonormal basis in $\mathbb{C}^{2 S+1}$ we have

$$
X_{n}^{T}=\sum_{j=-S}^{S} c_{n, j} V_{j}^{T}, \quad\left|X_{k}\right|^{2}=\sum_{j=-S}^{S}\left|c_{k, j}\right|^{2} .
$$

Using this in (71) we get

$$
\begin{aligned}
R^{T}\left(\lambda_{n}\right) & =\left(\lambda_{n} I-A\right) X_{n}^{T} \\
& =\sum_{j=-S}^{S}\left(\lambda_{n} c_{n, j} V_{j}^{T}-A\left(c_{n, j} V_{j}^{T}\right)\right) \\
& =\sum_{j=-S}^{S} c_{n, j}\left(\lambda_{n}-\mu_{j}\right) V_{j}^{T} .
\end{aligned}
$$

Multiplying both sides by $V_{j}^{T}$ we obtain

$$
c_{n, j}\left(\lambda_{n}-\mu_{j}\right)=\left\langle R^{T}\left(\lambda_{n}\right), V_{j}^{T}\right\rangle .
$$


On the other hand using the definition $R^{T}\left(\lambda_{n}\right),(82)$, and (88) we get

$$
\left|\left\langle R^{T}\left(\lambda_{n}\right), V_{j}^{T}\right\rangle\right| \leq 4 s c \varepsilon \frac{c^{r}}{(2 l)^{r}}=8 s l \varepsilon^{2}
$$

for all $n, j=0, \pm 1, \pm 2, \ldots, \pm l$. This with (93) implies (89).

By Schwarz inequality and (83) we have

$$
\left|\left\langle R^{T}\left(\lambda_{n}\right), V_{j}^{T}\right\rangle\right| \leq 2 c \varepsilon \sqrt{s}
$$

for all $n=0, \pm 1, \pm 2, \ldots, \pm l$ and $j=0, \pm 1, \pm 2, \ldots, \pm S$. Therefore (90) follows from (93).

Introduce the notation

$$
\begin{gathered}
Y_{n}=\left(\cdots X_{n,-S-1}, X_{n,-S}, X_{n,-S+1}, \ldots, X_{n, S}, X_{n, S+1}, \ldots\right), \\
U_{j}=\left(\cdots 0,0, V_{j,-S}, V_{j,-S+1}, \ldots, V_{j, S}, 0,0, \ldots\right) .
\end{gathered}
$$

Here $Y_{n}$ and $U_{j}$ are elements of $l_{2}$, and

$$
\left\langle Y_{n}, U_{j}\right\rangle=\sum_{i=-\infty}^{\infty} X_{n, i} \overline{V_{j, i}}=\sum_{i=-S}^{S} X_{n, i} \overline{V_{j, i}}=\left\langle X_{n}^{T}, V_{j}^{T}\right\rangle=c_{n, j} .
$$

Using equality (35) and the definition of $Y_{n}$ and $U_{j}$ one can easily verify that $\left\{Y_{n}: n=0, \pm 1, \pm 2, \ldots, \pm S\right\}$ and $\left\{U_{n}: n=\right.$ $0, \pm 1, \pm 2, \ldots, \pm S\}$ are the orthonormal systems in $l_{2}$.

Now we are ready to prove the following main result.

Theorem 6. If $l>\max \left\{c^{2}, 2 c, 3 s\right\}$ then the inequality

$$
\left|\lambda_{n}-\mu_{n}\right| \leq \frac{8 S s c^{2 r+2}}{(2 l)^{2 r+1}}
$$

holds for all $n=0, \pm 1, \pm 2, \ldots, \pm l$, where $S, r, l$ and $c, s$ are defined in (65) and (19).

Proof. Suppose to the contrary and without loss of generality that (98) does not hold for some $0 \leq n \leq l$. Then either $\lambda_{n}<$ $\mu_{n}-\left(8 S s c^{2 r+2} /(2 l)^{2 r+1}\right)$ or $\lambda_{n}>\mu_{n}+\left(8 S s c^{2 r+2} /(2 l)^{2 r+1}\right)$. Let us consider the case $\lambda_{n}<\mu_{n}-\left(8 S s c^{2 r+2} /(2 l)^{2 r+1}\right)$. Then

$$
\lambda_{k}<\mu_{j}-\frac{8 S s c^{2 r+2}}{(2 l)^{2 r+1}}
$$

and hence by (89) $\left|c_{k, j}\right|<1 / 2 S$ for all $k=0, \pm 1, \pm 2, \ldots$, $\pm n, j=n, \pm(n+1), \pm(n+2) \ldots, \pm l$. It implies that

$$
\left|c_{k, n}\right|^{2}+\sum_{j: n<|j| \leq l}\left|c_{k, j}\right|^{2} \leq \frac{2 l+1-2 n}{4 S^{2}}
$$

for $k=0, \pm 1, \pm 2, \ldots, \pm n$. On the other hand from Parseval's equality (91) we have

$$
\sum_{k=-n}^{n}\left|X_{k}\right|^{2}=\sum_{k=-n}^{n} \sum_{j=-S}^{S}\left|c_{k, j}\right|^{2}
$$

Now we are going to get a contradiction by proving that the left-hand side of (101) is greater than the right-hand side of (101). Using (84), the definition of $\delta$, and the conditions on $l$ one can easily verify that

$$
\sum_{k=-n}^{n}\left|X_{k}\right|^{2} \geq 2 n+1-(2 n+1) \delta>2 n+\frac{3}{4} .
$$

To estimate the right-hand side of (101) we write it as $S_{1}+S_{2}+$ $S_{3}$, where

$$
\begin{gathered}
S_{1}=\sum_{k=-n}^{n}\left(\left|c_{k, n}\right|^{2}+\sum_{j: n<|j| \leq l}\left|c_{k, j}\right|^{2}\right), \\
S_{2}=\sum_{k=-n}^{n} \sum_{j=-n}^{n-1}\left|c_{k, j}\right|^{2}, \quad S_{3}=\sum_{k=-n}^{n}\left(\sum_{j:|j|>l}\left|c_{k, j}\right|^{2}\right) .
\end{gathered}
$$

Using (100) and taking into account that $(2 l+1-2 n)+(2 n+1) \leq$ $2 S$ and hence $(2 l+1-2 n)(2 n+1) \leq S^{2}$ we obtain

$$
S_{1} \leq \frac{(2 l+1-2 n)(2 n+1)}{4 S^{2}}<\frac{1}{4} .
$$

Now let us estimate $S_{3}$. Using (99), (69), and then the inequality $l>2 c$ we obtain

$$
\left|\lambda_{k}-\mu_{j}\right|>\left|\mu_{l}-\mu_{j}\right|>|j|
$$

for $k=0, \pm 1, \pm 2, \ldots, \pm n$ and $|j|>l$. Therefore this, (90), and the definition $\varepsilon$ imply that

$$
\begin{aligned}
S_{3} & =\sum_{k=-n}^{n}\left(\sum_{j:|j|>l}\left|c_{k, j}\right|^{2}\right) \\
& \leq(2 n+1) \sum_{j:|j|>l}\left(\frac{2 c \varepsilon \sqrt{s}}{j}\right)^{2} \\
& <(2 n+1) \frac{4 s c^{2} \varepsilon^{2}}{l}<\frac{1}{4} .
\end{aligned}
$$

Now let us estimate $S_{2}$. Using (97) and the Bessel inequality for the elements $U_{i}$ for $i=-n,-n+1, \ldots, n-1$ with respect to the orthonormal systems $\left\{Y_{n}: n=0, \pm 1, \pm 2, \ldots, \pm n\right\}$ of $l_{2}$ we obtain

$$
\sum_{k=-n}^{n}\left|c_{k, i}\right|^{2} \leq\left|U_{i}\right|^{2}=1, \quad S_{2}=\sum_{i=-n}^{n-1} \sum_{k=-n}^{n}\left|c_{k, i}\right|^{2} \leq 2 n .
$$

The inequalities (104)-(107) show that the right side of (101) is less than $2 n+(1 / 2)$, which contradicts (102). In the same way we investigate the case $\lambda_{n}>\mu_{n}+\left(8 S s c^{2 r+2} /(2 l)^{2 r+1}\right)$. The theorem is proved.

\section{Examples and Conclusion}

In this section we illustrate the results of Sections 2 and 3 for the following examples. Let the potential $p_{s}(x)$ for $s=1,2,3$ of the operator $T\left(p_{s}\right)$ have the form

$$
p_{s}(x)=\sum_{n=1}^{s}\left(e^{i 2 n x}+e^{-i 2 n x}\right)=\sum_{n=1}^{s} 2 \cos 2 n x
$$


TABLE 1: Estimations for $T\left(p_{1}\right)$.

\begin{tabular}{lccc}
\hline & $x_{n, 3}$ & $E_{n, 3}$ & $\gamma_{n}$ \\
\hline$n=7$ & 49.0119073043627 & 0.00401827341683563 & 0.00803652968036530 \\
$n=8$ & 64.0090356900908 & 0.00232226049016466 & 0.00464451589853519 \\
$n=9$ & 81.0070967373201 & 0.00146120590904089 & 0.00292241001412498 \\
$n=10$ & 100.005724155838 & 0.00097836132370372 & 0.00195672191528545 \\
$n=20$ & 400.001412301984 & $8.57660779334148 \times 10^{-5}$ & 0.00017153215300668 \\
$n=30$ & 900.000626190365 & $2.27805363772165 \times 10^{-5}$ & $4.55610726195539 \times 10^{-5}$ \\
$n=40$ & 1600.00035193858 & $9.11289409047171 \times 10^{-6}$ & $1.82257881647412 \times 10^{-5}$ \\
$n=50$ & 2500.00022515394 & $4.5213654576927 \times 10^{-6}$ & $9.04273091219341 \times 10^{-6}$ \\
$n=60$ & 3600.00015632421 & $2.56272510680566 \times 10^{-6}$ & $5.12545021275656 \times 10^{-6}$ \\
$n=70$ & 4900.00011483597 & $1.59021161389524 \times 10^{-6}$ & $3.18042322750835 \times 10^{-6}$ \\
$n=80$ & 6400.0000879141 & $1.05364682405463 \times 10^{-6}$ & $2.10729364800086 \times 10^{-6}$ \\
$n=90$ & 8100.0000694591 & $7.33717636691826 \times 10^{-7}$ & $1.46743527333693 \times 10^{-6}$ \\
$n=100$ & 10000.0000562596 & $5.31248113844258 \times 10^{-7}$ & $1.06249622766647 \times 10^{-6}$ \\
\hline
\end{tabular}

TABle 2: Estimations for $T\left(p_{2}\right)$.

\begin{tabular}{lccc}
\hline & $x_{n, 3}$ & $E_{n, 3}$ & $\gamma_{n}$ \\
\hline$n=13$ & 169.006553875546 & 0.00801822430426367 & 0.01603644646924830 \\
$n=14$ & 196.005629484083 & 0.00602192413268590 & 0.01204384713096120 \\
$n=15$ & 225.004888933687 & 0.00463706113393842 & 0.00927412163367219 \\
$n=16$ & 256.004286247051 & 0.00364633797625785 & 0.00729267558174552 \\
$n=17$ & 289.003789043447 & 0.00291892692052321 & 0.00583785361582058 \\
$n=18$ & 324.003373962035 & 0.00237284215158748 & 0.00474568416174256 \\
$n=19$ & 361.003023794203 & 0.00195492184884340 & 0.00390984360625575 \\
$n=20$ & 400.002725629827 & 0.00162966444959707 & 0.00325932883855288 \\
$n=30$ & 900.001203843083 & 0.00040657655861401 & 0.00081315311464415 \\
$n=40$ & 1600.00067569654 & 0.00015796542624476 & 0.00031593085219360 \\
$n=50$ & 2500.00043201403 & $7.70627570578593 \times 10^{-5}$ & 0.00015412551405901 \\
$n=60$ & 3600.00029984729 & $4.32014439412344 \times 10^{-5}$ & $8.64028878675565 \times 10^{-5}$ \\
$n=70$ & 4900.00022022411 & $2.66004761436181 \times 10^{-5}$ & $5.32009522823765 \times 10^{-5}$ \\
$n=80$ & 6400.00016857341 & $1.75241067230997 \times 10^{-5}$ & $3.50482134443501 \times 10^{-5}$ \\
$n=90$ & 8100.00013317449 & $1.21491644560633 \times 10^{-5}$ & $2.42983289113352 \times 10^{-5}$ \\
$n=100$ & 10000.0001078601 & $8.76569198293195 \times 10^{-6}$ & $1.75313839654927 \times 10^{-5}$ \\
\hline
\end{tabular}

TABLE 3: Estimations for $T\left(p_{3}\right)$.

\begin{tabular}{lccc}
\hline & $x_{n, 3}$ & $E_{n, 3}$ & $\gamma_{n}$ \\
\hline$n=19$ & 361.004488989457 & 0.012018209724884 & 0.024036418816389 \\
$n=20$ & 400.004042369632 & 0.00990095572697077 & 0.0198019110415735 \\
$n=30$ & 900.001776635742 & 0.00230548774143664 & 0.00461097546712649 \\
$n=40$ & 1600.00099552468 & 0.00086829674995455 & 0.00173659349819394 \\
$n=50$ & 2500.00063601104 & 0.00041615591363515 & 0.00083231182695096 \\
$n=60$ & 3600.00044125198 & 0.00023064366100756 & 0.00046128732193269 \\
$n=70$ & 4900.00032399850 & 0.00014088338405301 & 0.00028176676807951 \\
$n=80$ & 6400.00024796876 & $9.22660434495073 \times 10^{-5}$ & 0.00018453208688902 \\
$n=90$ & 8100.00019587583 & $6.36768120642807 \times 10^{-5}$ & 0.00012735362412432 \\
$n=100$ & 10000.0001586304 & $4.57782012510395 \times 10^{-5}$ & $9.15564025001002 \times 10^{-5}$ \\
\hline
\end{tabular}


TABle 4: Approximation of eigenvalues.

\begin{tabular}{|c|c|c|c|}
\hline & $p_{1}$ & $p_{2}$ & $p_{3}$ \\
\hline$\overline{\lambda_{0}}$ & -0.455138604105 & -0.451676027152 & -0.4539320948685 \\
\hline$\lambda_{-1}$ & -0.110248816992 & -0.040158274572 & -0.0204737818081 \\
\hline$\lambda_{1}$ & 1.859108072514 & 1.4456177812459 & 1.3907354889190 \\
\hline$\lambda_{-2}$ & 3.917024772994 & 2.8976658743702 & 2.9541319115098 \\
\hline$\lambda_{2}$ & 4.371300982731 & 5.1886431499537 & 4.8580498527548 \\
\hline$\lambda_{-3}$ & 9.047739259808 & 8.9161585304864 & 7.9082824512658 \\
\hline$\lambda_{3}$ & 9.078368847202 & 9.4153327308285 & 10.2941738497520 \\
\hline$\lambda_{-4}$ & 16.032970081406 & 16.0004107071615 & 15.9213717462580 \\
\hline$\lambda_{4}$ & 16.033832340360 & 16.1585649096071 & 16.3957158213096 \\
\hline$\lambda_{-5}$ & 25.020840823290 & 25.0389311983095 & 24.9848629686203 \\
\hline$\lambda_{5}$ & 25.020854345449 & 25.0538295076160 & 25.1789211080558 \\
\hline$\lambda_{-6}$ & 36.014289910633 & 36.0293767228453 & 36.0144251509371 \\
\hline$\lambda_{6}$ & 36.014290046045 & 36.0319035321757 & 36.0877507661928 \\
\hline$\lambda_{-7}$ & 49.010418249424 & 49.0218195042565 & 49.0311600838136 \\
\hline$\lambda_{7}$ & 49.010418250365 & 49.0219701639618 & 49.0394601884444 \\
\hline$\lambda_{-8}$ & 64.007937189247 & 64.0164674336750 & 64.0248999242659 \\
\hline$\lambda_{8}$ & 64.007937189258 & 64.0164851040169 & 64.0271961781896 \\
\hline$\lambda_{-9}$ & 81.006250326633 & 81.0128685694864 & 81.0198291868669 \\
\hline$\lambda_{9}$ & 81.006250326634 & 81.0128693419217 & 81.0203601164022 \\
\hline$\lambda_{-10}$ & 100.005050675157 & 100.010339593273 & 100.015987594137 \\
\hline$\lambda_{10}$ & 100.005050675158 & 100.010339662550 & 100.016034084442 \\
\hline$\lambda_{-20}$ & 400.001253135321 & 400.002520531313 & 400.003809046181 \\
\hline$\lambda_{20}$ & 400.001253135326 & 400.002520531318 & 400.003809046182 \\
\hline$\lambda_{-30}$ & 900.000556173742 & 900.001115142518 & 900.001678193187 \\
\hline$\lambda_{30}$ & 900.000556173751 & 900.001115142519 & 900.001678193192 \\
\hline$\lambda_{-40}$ & 1600.00031269547 & 1600.00062627292 & 1600.00094113218 \\
\hline$\lambda_{40}$ & 1600.00031269548 & 1600.00062627292 & 1600.00094113219 \\
\hline$\lambda_{-50}$ & 2500.00020008004 & 2500.00040052089 & 2500.00060148494 \\
\hline$\lambda_{50}$ & 2500.00020008004 & 2500.00040052089 & 2500.00060148495 \\
\hline$\lambda_{-60}$ & 3600.00013892748 & 3600.00027802883 & 3600.00041738205 \\
\hline$\lambda_{60}$ & 3600.00013892749 & 3600.00027802885 & 3600.00041738205 \\
\hline$\lambda_{-70}$ & 4900.00010206165 & 4900.00020421710 & 4900.00030650836 \\
\hline$\lambda_{70}$ & 4900.00010206165 & 4900.00020421711 & 4900.00030650836 \\
\hline$\lambda_{-80}$ & 6400.00007813720 & 6400.00015632939 & 6400.00023460110 \\
\hline$\lambda_{80}$ & 6400.00007813721 & 6400.00015632940 & 6400.00023460110 \\
\hline$\lambda_{-90}$ & 8100.00006173602 & 8100.00012350634 & 8100.00018532630 \\
\hline$\lambda_{90}$ & 8100.00006173602 & 8100.00012350635 & 8100.00018532634 \\
\hline$\lambda_{-100}$ & 10000.00005000500 & 10000.00010003250 & 10000.00015009260 \\
\hline$\lambda_{100}$ & 10000.00005000500 & 10000.00010003260 & 10000.00015009260 \\
\hline
\end{tabular}

that is, $q_{n}=q_{-n}=1$ for $1 \leq n \leq s$ and $q_{n}=q_{-n}=0$ for $n>s$, where $q_{n}$ is defined in (9). Note that the operator $T\left(p_{1}\right)$ is a famous Mathieu operator. By (19) and (108), $Q=1$ and $M=$ $c$. For $s=1,2,3$ the constant $M$ or $c$ has the values of 2, 4, 6, respectively. The fixed point approximations $x_{n, 3}$ determined in (55), where $f(x)$ is defined by (40) with $m=3$, of the eigenvalues $\lambda_{ \pm n}$ of the operators $T\left(p_{s}\right)$ for $s=1,2,3$ are given in Tables 1, 2, and 3, respectively. Moreover, the estimations of the error $E_{n, 3}=\left|\lambda_{ \pm n}-x_{n, 3}\right|$ (see (64)) and the length $\gamma_{n}$ of the $n$th gap (see (45)) are also given in Tables 1,2 , and 3 .

The method of Section 3 gives high precision results for the calculation of the small eigenvalues. Let us illustrate it by using formula (98) for the first 201 eigenvalues $\lambda_{0}, \lambda_{-1}, \lambda_{1}, \lambda_{-2}, \lambda_{2}, \ldots, \lambda_{-100}, \lambda_{100}$ of the operators $T\left(p_{s}\right)$ for $s=1,2,3$. It means that the number $l$ in (98) is 100 (see the first sentence of Section 3). To find an approximation with error of order $10^{-18}$ for the eigenvalues of $T\left(p_{1}\right)$ we take $r=5$. Therefore for the potential $p_{s}(x)$, where $s=$ $1,2,3$, the number $S$ is $l+2 r s=100+10 s$ and the number of equations in (65) is $2 S+1=200+20 s+$ 1. The matrices of (65) corresponding to the potentials $p_{1}(x), p_{2}(x), p_{3}(x)$ and denoted by $A_{1}, A_{2}, A_{3}$ are of order 221, 241, and 261, respectively. The approximate eigenvalues $\mu_{0}, \mu_{-1}, \mu_{1}, \mu_{-2}, \mu_{2}, \ldots, \mu_{-100}, \mu_{100}$ of the matrices $A_{1}, A_{2}, A_{3}$ are given in Table 4 . By (98) the eigenvalues $\mu_{n}$ are very close to the eigenvalues $\lambda_{n}$ of the operator $T\left(p_{s}\right)$. One can readily see from (98) that the approximation $\left|\lambda_{n}-\mu_{n}\right|$ of $\lambda_{n}$ by the eigenvalues $\mu_{n}$ is arbitrary small if $r$ is a large number and $c$ is 
TABLE 5: Approximation of the lengths of the gaps.

\begin{tabular}{lccc}
\hline & $p_{1}$ & $p_{2}$ & $p_{3}$ \\
\hline$\gamma_{1}$ & 1.96935688950626 & 1.48577605581811 & 1.41120927072708 \\
$\gamma_{2}$ & 0.45427620973738 & 2.29097727558352 & 1.90391794124493 \\
$\gamma_{3}$ & 0.03062958739405 & 0.49917420034206 & 2.38589139848613 \\
$\gamma_{4}$ & 0.00086225895372 & 0.15815420244566 & 0.47434407505152 \\
$\gamma_{5}$ & $1.35221586674561 \times 10^{-5}$ & 0.01489830930653 & 0.19405813943552 \\
$\gamma_{6}$ & $1.35412271617952 \times 10^{-7}$ & 0.00252680933036 & 0.07332561525572 \\
$\gamma_{7}$ & $9.41085431804822 \times 10^{-10}$ & 0.00015065970523 & 0.00830010463081 \\
$\gamma_{8}$ & $1.09992015495664 \times 10^{-11}$ & $1.76703419043633 \times 10^{-5}$ & 0.00229625392370 \\
$\gamma_{9}$ & $5.82645043323282 \times 10^{-13}$ & $7.72435271301219 \times 10^{-7}$ & 0.00053092953526 \\
$\gamma_{10}$ & $1.22213350550737 \times 10^{-12}$ & $6.92769646093439 \times 10^{-8}$ & $4.64903052659338 \times 10^{-5}$ \\
$\gamma_{20}$ & $5.11590769747272 \times 10^{-12}$ & $4.584747350886464 \times 10^{-13}$ & $9.09494701772928 \times 10^{-13}$ \\
$\gamma_{30}$ & $3.64019966684282 \times 10^{-12}$ & $3.63797880709171 \times 10^{-12}$ & $4.43378667114303 \times 10^{-12}$ \\
$\gamma_{40}$ & $3.41060513164848 \times 10^{-12}$ & $6.82121026329696 \times 10^{-12}$ & $1.02318153949454 \times 10^{-11}$ \\
$\gamma_{50}$ & $3.18323145620525 \times 10^{-12}$ & $1.90993887372315 \times 10^{-11}$ & $5.45696821063757 \times 10^{-12}$ \\
$\gamma_{60}$ & $7.27595761418343 \times 10^{-12}$ & $5.45696821063757 \times 10^{-12}$ & $4.09272615797818 \times 10^{-12}$ \\
$\gamma_{70}$ & $3.63797880709171 \times 10^{-12}$ & $5.45696821063757 \times 10^{-12}$ & $2.72848410531878 \times 10^{-12}$ \\
$\gamma_{80}$ & $6.3664629124105 \times 10^{-12}$ & $6.3664629124105 \times 10^{-12}$ & $1.81898940354586 \times 10^{-12}$ \\
$\gamma_{90}$ & $1.81898940354586 \times 10^{-12}$ & $2.91038304567337 \times 10^{-11}$ & $4.09272615797818 \times 10^{-11}$ \\
$\gamma_{100}$ & $1.09139364212751 \times 10^{-11}$ & 0 & 0 \\
\hline
\end{tabular}

a small number. If the potential $q$ is smooth function, then the number $c$ is a small number (see (13) and (19)), and hence (98) gives better approximations for smooth potentials. Moreover if $s$ is a small number, that is, the number of summand of $p_{s}$ (see (108)) is small, then we can choose $r$ so that the order of the matrix $A_{s}$ is not a large number while the approximation (98) is a very small number. By formula (98) $\left|\lambda_{n}-\mu_{n}\right|$, where $n=0, \pm 1, \pm 2, \ldots, \pm 100$, for the potentials $p_{1}(x), p_{2}(x)$, and $p_{3}(x)$ is not greater than

$$
\begin{gathered}
\frac{8 \times 110 \times 2^{12}}{(200)^{11}}=\frac{11}{625} 10^{-17}, \\
\frac{8 \times 120 \times 2 \times 4^{12}}{(200)^{11}}=\frac{3}{1907348632812500}, \\
\frac{8 \times 130 \times 3 \times 6^{12}}{(200)^{11}}=\frac{20726199}{62500000000000000000},
\end{gathered}
$$

respectively. Thus in Section 3 there are the following observations to be considered. Instead of the matrices of order 201 investigating a little big matrices, namely, matrices of order 221,241, and 261, we find an approximation of order $10^{-18}, 10^{-15}$, and $10^{-12}$ for the first 201 eigenvalues of $T\left(p_{1}\right), T\left(p_{2}\right)$, and $T\left(p_{3}\right)$, respectively. Moreover this approach is applicable for the trigonometric polynomial potentials and for the sufficiently differentiable periodic potentials.

The estimations of the lengths $\gamma_{1}, \gamma_{2}, \ldots, \gamma_{100}$ of the gaps are given in Table 5. It is known that [12] for large $n$ the behavior of $\gamma_{n}$ is sensitive to smoothness properties of the potential $q$. If $q$ is $m$ times differentiable, then $\gamma_{n}=O\left(n^{-m}\right)$. If $q$ is analytic function, then $\gamma_{n}=O\left(e^{-a n}\right)$ for some positive a. For the Mathieu operator $T\left(p_{1}\right)$ the following asymptotic formula holds: $\gamma_{n}=O\left(4^{n} /((n-1) !)^{2}\right)$. Thus for large $n$ the length $\gamma_{n}$ of the $n$th gap is a very small number. Table 5 confirms this result for large $n$ (see $\gamma_{n}$ for $n \geq 10$ ). Moreover Table 5 shows that these results continue to hold for $n>$ 5. Since for the small values of $n(n \leq 5)$ the asymptotic formulas do not give any information, we cannot compare the theoretical results with the results in Table 5. Note that in Tables 4 and 5 the eigenvalues and the lengths of the gaps are computed using Matlab. In Table 4 this program transects to 14 figures, because this accuracy is acceptable for estimations of the eigenvalues. However, we compute the lengths of the gaps without transaction, since (as it is noted above) for large $n$ the theoretical results give the estimations of $\gamma_{n}$ with very high accuracy.

It is natural and well known that for large eigenvalues the asymptotic method gives us approximations with smaller errors. Since the method of Section 3 gives high precision results for the small eigenvalues and gaps (see Tables 4 and 5 ), the comparison of the Tables 1-5, where we estimate the eigenvalues and gaps by the methods of Sections 2 and 3, respectively, for the potential (108), shows that the results of the asymptotic method given in Tables 1-3 are not precise for the small eigenvalues.

\section{References}

[1] M. S. P. Eastham, The Spectral Theory of Periodic Differential Equations, Scottish Acedemic Press, Edinburg, UK, 1973.

[2] J. Pöschel and E. Trubowitz, Inverse Spectral Theory, Academic Press, Boston, Mass, USA, 1987.

[3] A. L. Andrew, "Correction of finite element eigenvalues for problems with natural or periodic boundary conditions," BIT, vol. 28 , no. 2, pp. 254-269, 1988. 
[4] A. L. Andrew, "Correction of finite difference eigenvalues of periodic Sturm-Liouville problems," Journal of Australian Mathematical Society B, vol. 30, no. 4, pp. 460-469, 1989.

[5] D. J. Condon, "Corrected finite difference eigenvalues of periodic Sturm-Liouville problems," Applied Numerical Mathematics, vol. 30, no. 4, pp. 393-401, 1999.

[6] G. Vanden Berghe, M. Van Daele, and H. De Meyer, "A modified difference scheme for periodic and semiperiodic SturmLiouville problems," Applied Numerical Mathematics, vol. 18, no. 1-3, pp. 69-78, 1995.

[7] X. Ji and Y. S. Wong, "Prüfer method for periodic and semiperiodic sturm-liouville eigenvalue problems," International Journal of Computer Mathematics, vol. 39, pp. 109-123, 1991.

[8] Y. S. Wong and X. Z. Ji, "On shooting algorithm for SturmLiouville eigenvalue problems with periodic and semi-periodic boundary conditions," Applied Mathematics and Computation, vol. 51, no. 2-3, pp. 87-104, 1992.

[9] X. Z. Ji, “On a shooting algorithm for Sturm-Liouville eigenvalue problems with periodic and semi-periodic boundary conditions," Journal of Computational Physics, vol. 111, no. 1, pp. 74-80, 1994.

[10] V. Malathi, M. B. Suleiman, and B. B. Taib, "Computing eigenvalues of periodic Sturm-Liouville problems using shooting technique and direct integration method," International Journal of Computer Mathematics, vol. 68, no. 1-2, pp. 119-132, 1998.

[11] O. A. Veliev and M. T. Duman, "The spectral expansion for a nonself-adjoint Hill operator with a locally integrable potential," Journal of Mathematical Analysis and Applications, vol. 265, no. 1, pp. 76-90, 2002.

[12] J. Avron and B. Simon, "The asymptotics of the gap in the Mathieu equation," Annals of Physics, vol. 134, no. 1, pp. 76-84, 1981. 


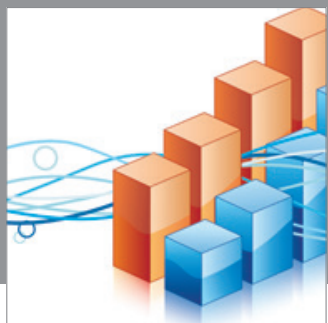

Advances in

Operations Research

mansans

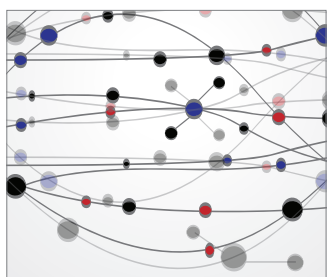

The Scientific World Journal
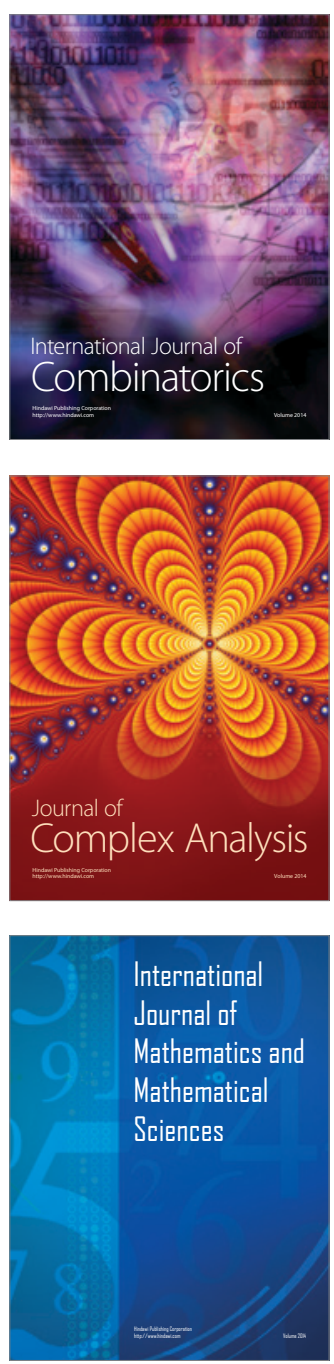
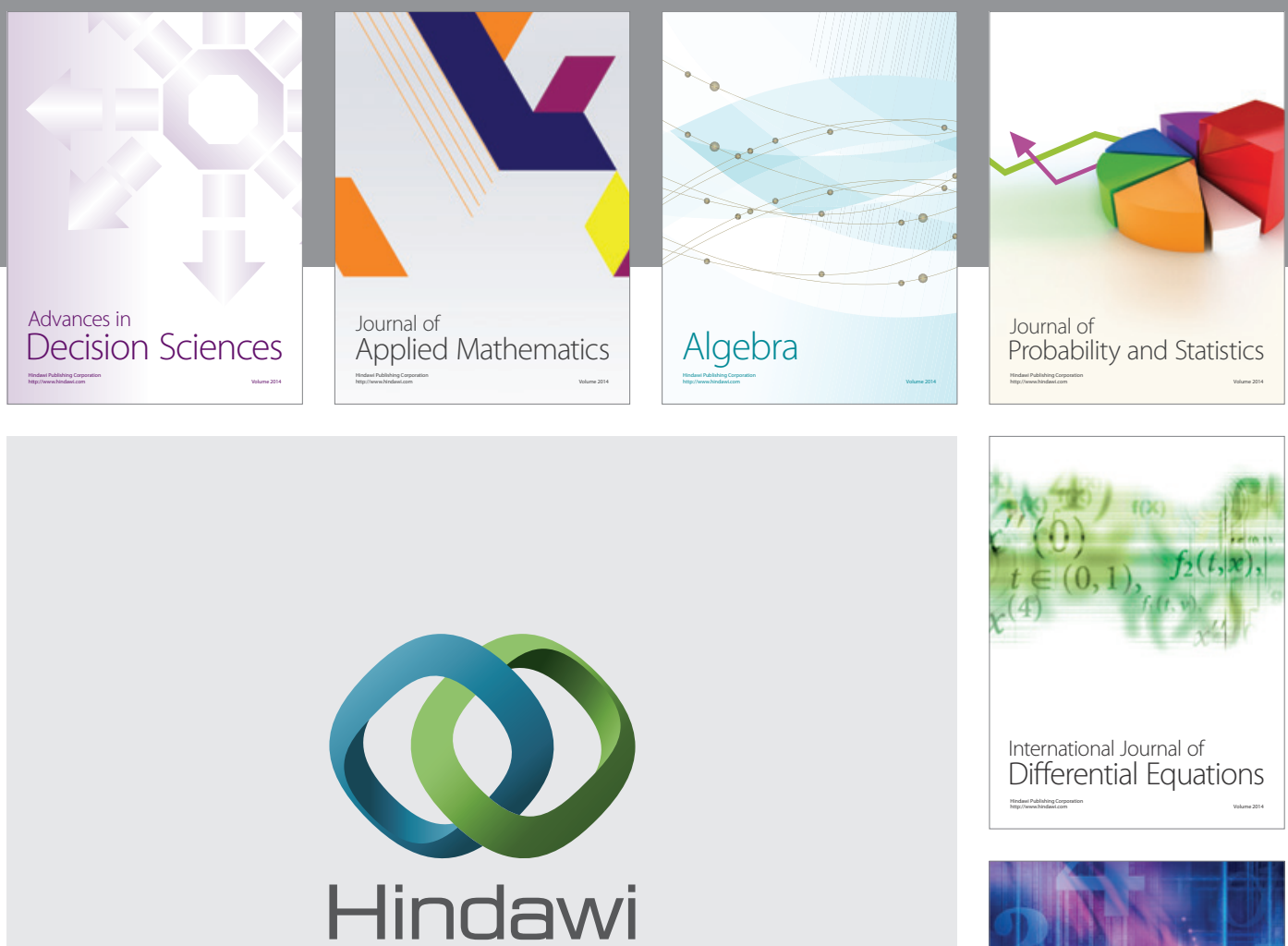

Submit your manuscripts at http://www.hindawi.com
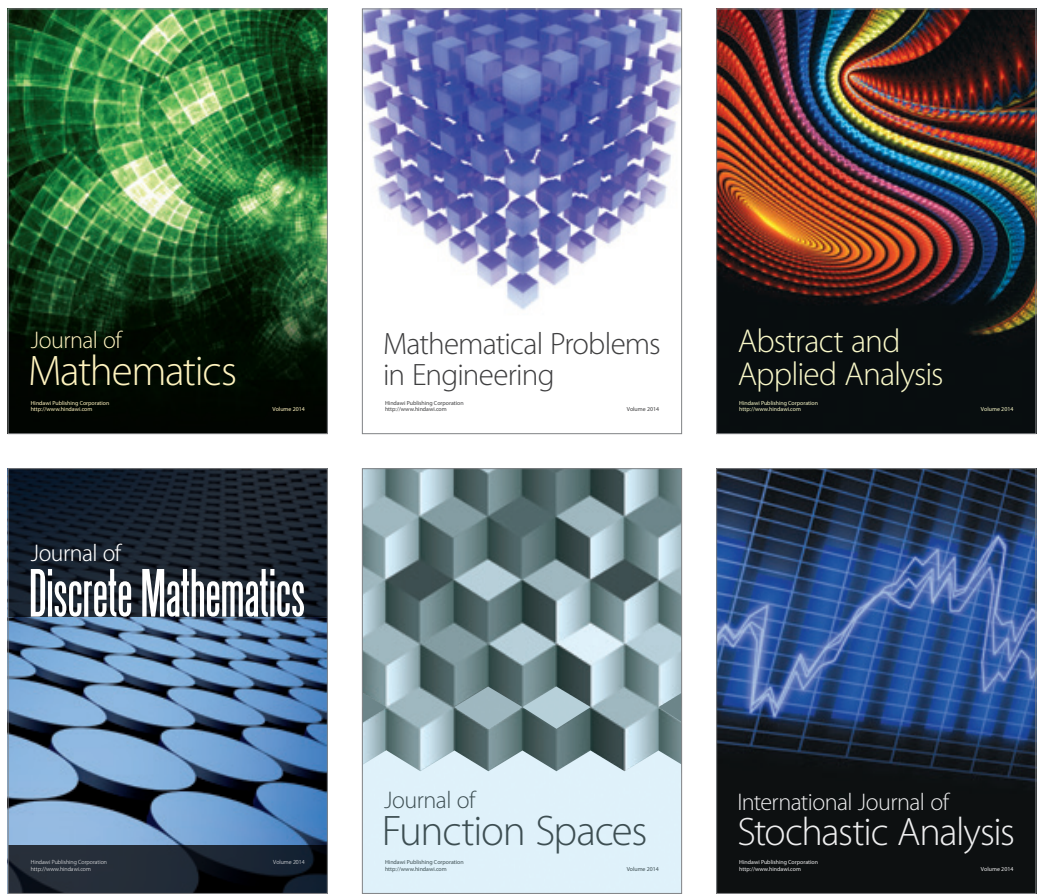

Journal of

Function Spaces

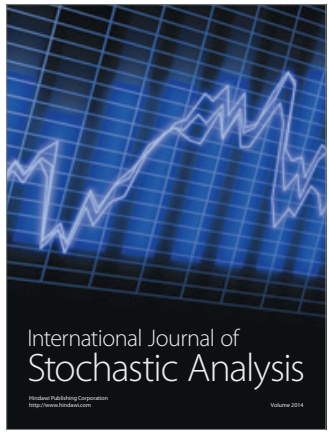

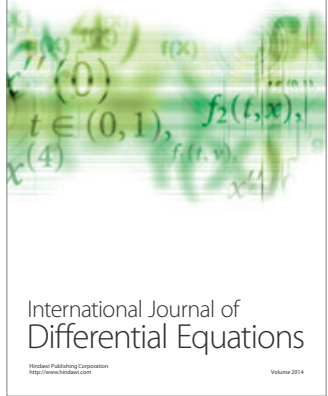
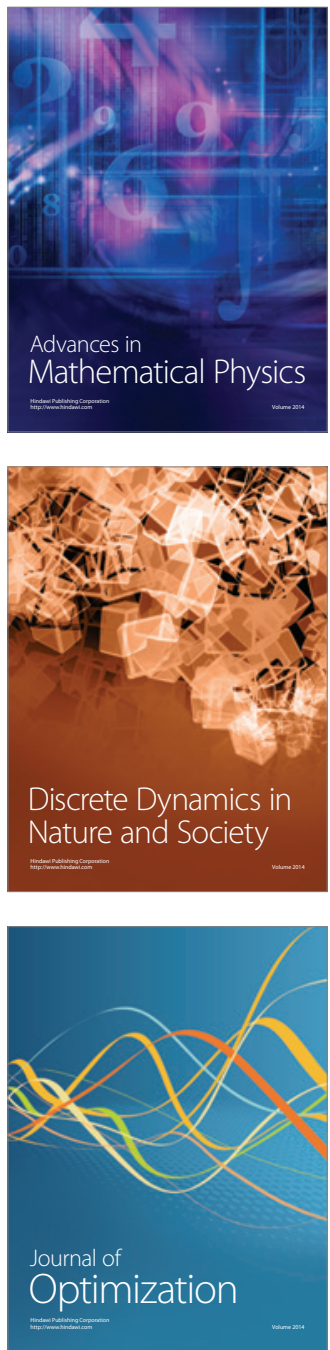